\title{
Improved Delineation of Parathyroid Lesions in Patients with Chronic Renal Failure Using Magnified Pinhole Imaging
}

\author{
Layla Ali ${ }^{1}$, Issa Loutfi ${ }^{2}$, Gautam Biswas ${ }^{3}$, Nadia Hadi $^{1}$, and Tamer Girgis ${ }^{3}$ \\ ${ }^{I}$ Department of Radiologic Sciences, Faculty of Allied Health Sciences, Kuwait University, Mishref, Kuwait; ${ }^{2}$ Department of Nuclear \\ Medicine, Faculty of Medicine, Kuwait University, Mishref, Kuwait; and ${ }^{3}$ Department of Nuclear Medicine, Hamed Al-Issa \\ Transplant Center, Ministry of Health, Safat, Kuwait
}

99mTc-sestamibi dual-phase scintigraphy is currently established for parathyroid localization. However, the imaging technique is not standardized, and the role of the pinhole collimator, especially, is not fully recognized in the imaging protocol. The aim of this study was to check whether the use of a pinhole collimator in parathyroid scintigraphy would enhance lesion detectability and delineation more than does a parallel-hole collimator or SPECT in patients with secondary hyperparathyroidism due to chronic renal failure with a mixed pattern of abnormalities. Methods: Thirty-five patients referred for a parathyroid scan were included. Imaging was performed at $10 \mathrm{~min}$ and $2 \mathrm{~h}$ after injection of $925 \mathrm{MBq}(25 \mathrm{mCi})$ of $99 \mathrm{mTc}$-sestamibi using both a pinhole collimator and a high-resolution parallelhole collimator fitted to a scintillation camera. SPECT was also performed at $1.5 \mathrm{~h}$ after injection. The images were reviewed by 2 experienced nuclear medicine physicians, and the results were analyzed. In addition, the contrast of visualized lesions was evaluated. Results: Twenty-three patients $(65.7 \%)$ had abnormal scan findings. The McNemar test revealed better detection of parathyroid lesions using pinhole imaging than with planar parallel-hole imaging and SPECT $(P<0.001$ and $P<$ 0.03 , respectively). Both observers showed good agreement in evaluating different imaging techniques $(\kappa=0.76)$. Observers were in favor of pinhole imaging because SPECT suffered from noise. Lesion contrast was significantly higher in pinhole imaging than in parallel-hole imaging and SPECT $(P<0.05)$, with a $16 \%$ and $11 \%$ improvement in contrast, respectively. Conclusion: Pinhole imaging better delineates and detects lesions in parathyroid scintigraphy than does parallel-hole imaging and SPECT. Pinhole imaging increases confidence in image interpretation because of high lesion contrast and better magnification and resolution. The use of this technique is therefore recommended as part of the routine imaging protocol for 99mTc-sestamibi parathyroid scintigraphy.

Key Words: hyperparathyroidism; ${ }^{99 m T c-s e s t a m i b i ; ~ p i n h o l e ~}$ collimator; renal failure

J Nucl Med Technol 2011; 39:35-39

DOI: 10.2967/jnmt.110.076984

Received Mar. 5, 2010; revision accepted Oct. 1, 2010.

For correspondence or reprints contact: Layla Ali, Department of Radiologic Sciences, Faculty of Allied Health Sciences, Kuwait University, P.O. Box 2604, Mishref 40177, Kuwait.

E-mail: layla@hsc.edu.kw

COPYRIGHT @ 2011 by the Society of Nuclear Medicine, Inc.
D ual-phase scintigraphy using ${ }^{99} \mathrm{~m}$ Tc-sestamibi is an established procedure for detection and localization of parathyroid lesions in patients in whom the diagnosis of hyperparathyroidism has been confirmed $(1-3)$.

The mechanism of ${ }^{99 \mathrm{~m}} \mathrm{Tc}$-sestamibi uptake is related to its chemical nature as a lipophilic cation diffusing passively into the cell and localizing more preferentially in the mitochondria. The main attribute of this agent in the context of parathyroid imaging is that it demonstrates slower washout from the functionally active parathyroid lesions than from adjacent thyroid tissue, thus permitting detection of parathyroid abnormalities by showing differential clearance over time- the so-called single-isotope dual-phase technique. In practice, early and late images are obtained after intravenous injection of the tracer, making this technique simple, easy to perform, and superior to the earlier technetium-thallium subtraction method $(1,2,4)$.

A review of ${ }^{99 \mathrm{~m}} \mathrm{Tc}$-sestamibi methodology, however, reveals that the imaging protocol varies and that the procedure has not been completely standardized $(4,5)$. The most commonly used sequence is early planar imaging (mostly using a parallel-hole collimator) at about $10 \mathrm{~min}$ and delayed imaging using the same technique at $2 \mathrm{~h}$ after injection of the radiopharmaceutical. SPECT is added in most protocols at $1-2 \mathrm{~h}$ after injection. To improve the detectability of lesions, some studies have used a pinhole collimator for imaging and reported better localization of adenomas than can be obtained with a parallel-hole collimator or delayed SPECT (6-8). A combination of early and delayed pinhole imaging and SPECT was also reported to maximize parathyroid lesion detection (9). Pinhole SPECT was shown to be useful as a complement to planar scintigraphy, especially in secondary hyperparathyroidism $(10,11)$. However, the complexity of setting up pinhole SPECT and, especially, of processing the images has precluded its use in routine practice.

Currently, challenges remain for optimal use of imaging techniques in parathyroid scintigraphy, especially for 
improving delineation of subtle parathyroid lesions such as small adenomas and hyperplasia in secondary hyperparathyroidism due to renal failure (12-14). In this study, the aim was to show whether the use of pinhole imaging in parathyroid scintigraphy would enhance lesion detectability and delineation more than would parallel-hole imaging or SPECT in patients with secondary hyperparathyroidism due to chronic renal failure, in which a mixed pattern of abnormalities (adenoma and hyperplasia) can occur.

\section{MATERIALS AND METHODS}

Thirty-five patients with chronic renal failure who were referred for a parathyroid scan but had not undergone neck surgery were included in this study. There were 13 male and 22 female patients. The age range was 18-70 y (mean, 43 y). All patients had elevated serum parathyroid hormone levels. The study was approved by the local ethics committee, and all patients gave their informed consent to participate in it. Imaging was performed using a Millennium dual-head camera (GE Healthcare) at $10 \mathrm{~min}$ and at $2 \mathrm{~h}$ after intravenous injection of $925 \mathrm{MBq}(25 \mathrm{mCi})$ of ${ }^{99 \mathrm{~m}} \mathrm{Tc}-$ sestamibi. The imaging protocol was as follows: At $10 \mathrm{~min}$ after injection, the patient was positioned supine on the imaging table with the neck fully extended. An anterior static image of the neck was acquired using a pinhole collimator (5-mm aperture size). The distance between the patient and the pinhole aperture was adjusted to center the thyroid in the field of view and optimally magnify the area of interest. The same distance was used for both early and delayed imaging. The camera was then mounted with a high-resolution parallel-hole collimator with zoom factor of 2.0. A second static image of the anterior neck and chest was then acquired for $10 \mathrm{~min}$. All static images were acquired using a $256 \times 256$ pixel matrix. At $2 \mathrm{~h}$ after injection, that is, directly after SPECT acquisition, static images using parallel-hole and pinhole collimators were acquired in the same way as for the early imaging.

In addition to planar imaging, SPECT was acquired at $1.5 \mathrm{~h}$ after injection of the radiopharmaceutical, as this time was found to provide good counting statistics and a fair washout of the activity from the thyroid gland. The acquisition parameters were 64 projections, $30 \mathrm{~s} /$ projection, a $360^{\circ}$ elliptic orbit, a $64 \times 64$ matrix, and a zoom of 1.67. Processing included filtered backprojection with a Butterworth filter cutoff frequency of 0.55 cycles/cm and a power of 10 .

The resulting images were reviewed by 2 experienced nuclear medicine physicians. Each observer scored the scans as negative or positive for parathyroid abnormalities (absence or presence of a focal area showing a prolonged or increased uptake over time). Image interpretation data were analyzed using the McNemar test, and interobserver agreement was assessed using the $\kappa$-value. For contrast measurement, one region of interest was drawn on the lesion and another of the same size on the background for the late static parallel-hole and pinhole images. For SPECT images, regions of interest were drawn on the 2 consecutive coronal slices that showed the highest tracer accumulation in parathyroid tissue. Lesion contrast was calculated for cases in which the 3 techniques showed positive results using the following formula: mean count in lesion - mean count in background/mean count in lesion. The results were analyzed using the Wilcoxon signed rank test.

\section{RESULTS}

Twenty-three patients $(65.7 \%)$ had abnormal scan findings according to at least 1 technique. In 17 patients (74\%),
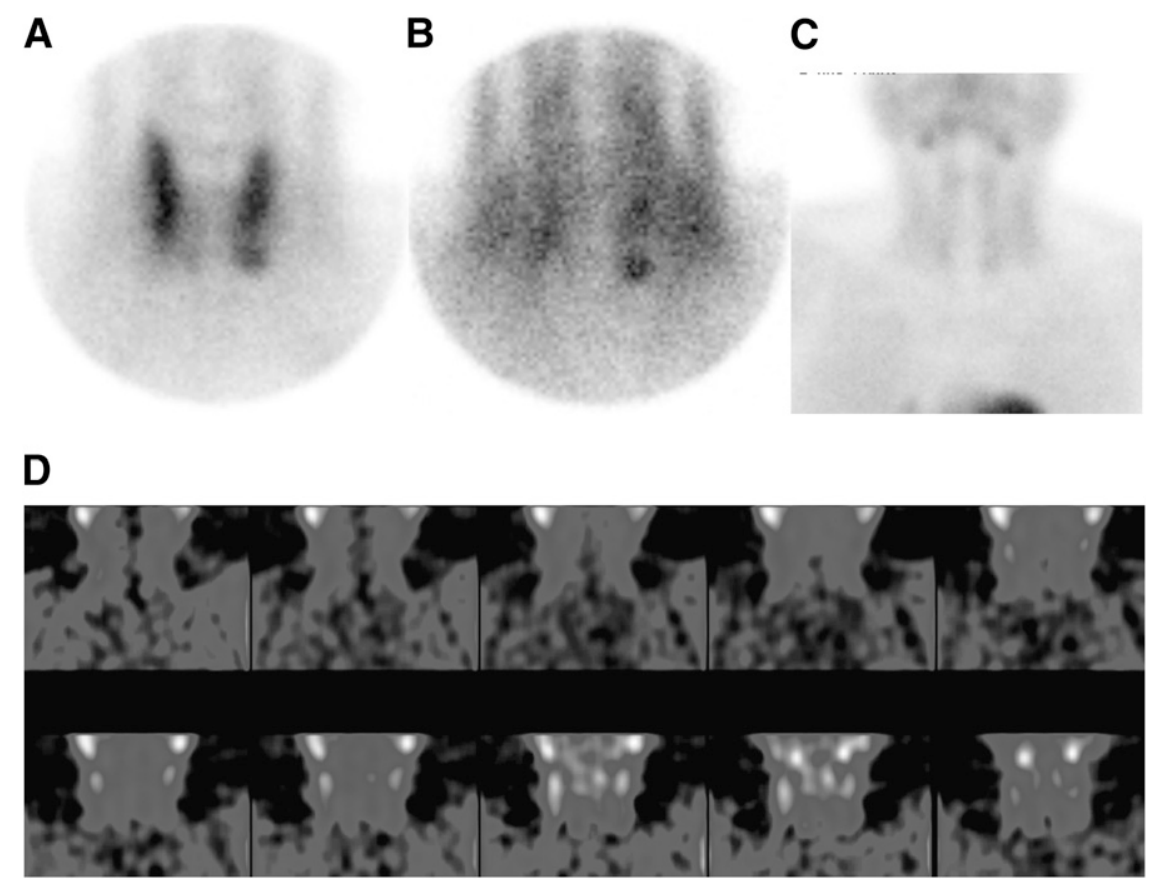

FIGURE 1. Lesion delineation in lower and middle left lobes was better on pinhole imaging at $10 \mathrm{~min}(\mathrm{~A})$ and $2 \mathrm{~h}(\mathrm{~B})$ than on parallel-hole imaging at $2 \mathrm{~h}(\mathrm{C})$, which had inconclusive findings. High noise level on SPECT images (D) precluded identification of parathyroid lesions. 
TABLE 1

McNemar Test Results for Comparison Between Pinhole and Parallel Collimators for Observer 1

\begin{tabular}{lccc}
\hline & \multicolumn{2}{c}{ Pinhole collimator } & \\
\cline { 2 - 3 } Parallel collimator & + & - & Total \\
\hline+ & 6 & 0 & 6 \\
- & 15 & 2 & 17 \\
Total & $21(91.3 \%)$ & $2(8.7 \%)$ & $P<0.001$ \\
\hline
\end{tabular}

more than 1 abnormality was seen (Figs. 1 and 2). The result of the comparison between pinhole images and parallel-hole or SPECT images using the McNemar test (Tables 1-4) revealed better detection of parathyroid lesions using the pinhole images (reviewer 1: $P<0.001$ for pinhole vs. high-resolution parallel-hole imaging and $P<0.03$ for pinhole imaging vs SPECT, Tables 1 and 2; reviewer 2: $P<0.001$ and $P<0.01$, respectively, Tables 3 and 4). Both observers showed substantial agreement in the way they interpreted the findings of the different imaging techniques tested $(\kappa=0.76)$. Lesion contrast was significantly higher in pinhole imaging than in parallel-hole imaging $\left(n=6, \mathrm{~W}=21, \sigma_{\mathrm{W}}= \pm 9.54, P<0.05\right)$ and SPECT $\left(n=6, \mathrm{~W}=19, \sigma_{\mathrm{W}}= \pm 9.54, P<0.05\right)$. The improvement in lesion contrast using the pinhole images was on the order of 16\% (range, 11\%-29\%) and 11\% (range, 8\%$17 \%$ ), compared with planar high-resolution parallel-hole imaging and SPECT, respectively. In patient studies for which both SPECT and pinhole images were positive, $25 \%$ of the lesions were better delineated with SPECT. On the other hand, SPECT underestimated the size, number, and uptake of $75 \%$ of lesions, compared with pinhole imaging, which clearly delineated parathyroid lesions (Fig. 2).

\section{DISCUSSION}

Our results show a clear advantage for pinhole imaging over parallel-hole imaging in the delineation and detection of parathyroid lesions in patients with secondary hyperparathyroidism due to chronic renal failure. The improved resolution and especially contrast of the lesions, even compared with SPECT, warrants the use of pinhole collimators as part of the routine imaging protocol for ${ }^{99 \mathrm{~m}} \mathrm{Tc}-$ sestamibi parathyroid scintigraphy. From a logistic and practical point of view, changing collimators from parallel-hole to pinhole and vice versa requires additional time

TABLE 2

McNemar Test Results for Comparison Between Pinhole Collimator and SPECT for Observer 1

\begin{tabular}{lccc}
\hline & \multicolumn{2}{c}{ SPECT } & \\
\cline { 2 - 3 } Pinhole collimator & + & - & Total \\
\hline+ & 9 & 11 & 20 \\
- & 2 & 1 & 3 \\
Total & $11(47.8 \%)$ & $12(52.2 \%)$ & $P<0.03$ \\
\hline
\end{tabular}

TABLE 3

McNemar Test Results for Comparison Between Pinhole and Parallel Collimators for Observer 2

\begin{tabular}{lccc}
\hline & \multicolumn{2}{c}{ Pinhole collimator } & \\
\cline { 2 - 3 } Parallel collimator & + & - & Total \\
\hline+ & 6 & 0 & 6 \\
- & 13 & 4 & 17 \\
Total & $19(82.6 \%)$ & $4(17.4 \%)$ & $P<0.001$ \\
\hline
\end{tabular}

and effort in a busy nuclear medicine department and can be impractical in a department with 1 or 2 cameras and only occasional referral for parathyroid scans. However, pinhole imaging is worthwhile because it can make the study far more accurate, potentially having a significant effect on subsequent management of the patient $(2,4,15)$. The benefits of using pinhole imaging that are highlighted by this study are 2-fold. The first is the increased contrast of the lesions seen, giving the interpreting physician more confidence in reporting abnormalities. The second is the better delineation of small lesions thanks to the ability of the pinhole collimator to magnify small lesions and to detect deep abnormalities, thus adding an element of enhanced resolution to the images. The addition of SPECT will also facilitate localization of the lesion by the inherent depth information and improved contrast, compared with planar imaging. This is in line with other studies that have shown that, compared with parallel imaging for preoperative parathyroid localization, SPECT increases the detection of parathyroid adenomas and the level of confidence in their detection (16). Despite the benefits of SPECT, our data show pinhole imaging to have definite advantages over SPECT. This finding was not a surprise because SPECT suffers from many factors that degrade the final reconstructed images. In our study, although an elliptic orbit was used for the acquisition, we found that some degradation in spatial resolution occurred because of the considerable distance between the neck of the patient and the face of the camera. In addition, the minor patient motion that is possible during an acquisition of about 32 min could further deteriorate SPECT image quality. Furthermore, the observers expressed a preference for pinhole images over parallel-hole images or SPECT, particularly because of the SPECT image noise that may lead to false-positive findings.

TABLE 4

McNemar Test Results for Comparison Between Pinhole Collimator and SPECT for Observer 2

\begin{tabular}{lccc}
\hline & \multicolumn{2}{c}{ SPECT } & \\
\cline { 2 - 3 } Pinhole collimator & + & - & Total \\
\hline+ & 11 & 11 & 22 \\
- & 1 & 0 & 1 \\
Total & $12(52 \%)$ & $11(48 \%)$ & $P<0.01$ \\
\hline
\end{tabular}


A

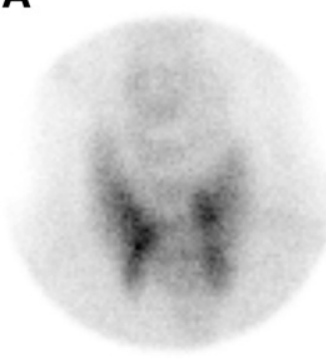

B

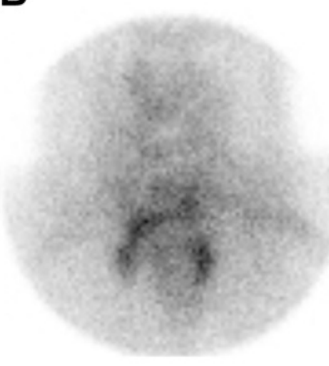

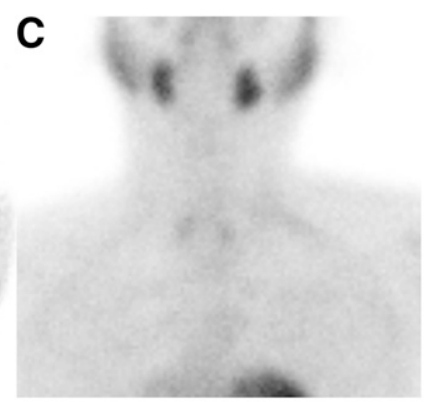

D

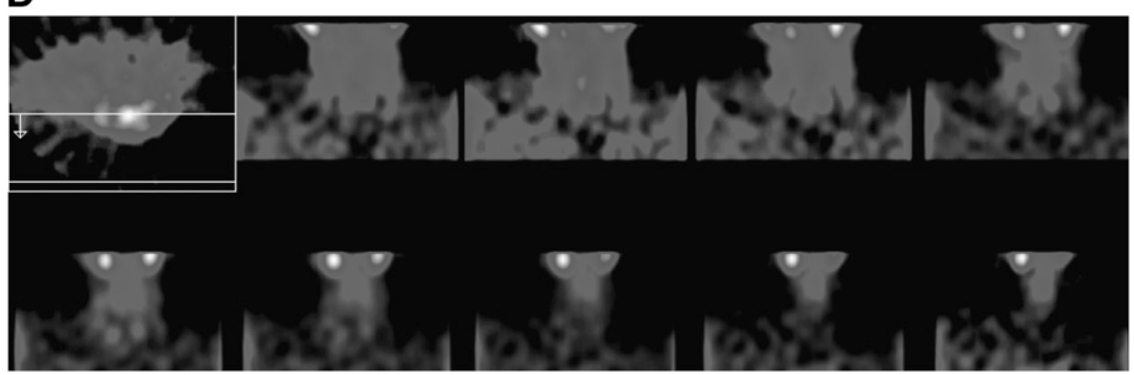

FIGURE 2. Four lesions are found on pinhole imaging (at $10 \mathrm{~min}[\mathrm{~A}]$ and $2 \mathrm{~h}$ [B]), but only 2 lesions are found on high-resolution parallel-hole imaging $(C)$ and SPECT (D). secondary hyperparathyroidism due to chronic renal failure. This type of imaging increases confidence in image interpretation because of high lesion contrast and better magnification and resolution. The use of this technique is therefore recommended as part of the routine imaging protocol for ${ }^{99 \mathrm{~m}} \mathrm{Tc}$-sestamibi parathyroid scintigraphy.

\section{ACKNOWLEDGMENTS}

We acknowledge the staff of the Nuclear Medicine Department in the transplant center for their collaboration and for conducting the clinical studies. We also thank Aqla Al-Mutairi for her technical assistance. the relatively poor counts collected from the abnormal parathyroids can lead to difficulties with SPECT reconstruction, which could add more noise to the parathyroid image. In contrast to high-resolution parallel-hole collimators, pinhole collimators are able to separate overlying structures since detection of photons emerging from different depths occurs at separate locations on the camera face. Also, pinhole collimators offer a higher magnification than the hardware zoom when the object is close to the pinhole, resulting in a better resolution for small lesions and a good sensitivity, compared with the highresolution parallel-hole collimator.

More recently, the introduction of SPECT/CT has been advocated to more accurately localize parathyroid lesions $(17,18)$. Further experience with SPECT/CT could show more benefits. The use of pinhole imaging would still be, however, the best approach for an improved delineation and detectability of parathyroid lesions, as we have demonstrated in this work.

\section{CONCLUSION}

Pinhole imaging in parathyroid scintigraphy leads to improved delineation of parathyroid lesions in patients with

\section{REFERENCES}

1. Coakley AJ, Kettle AG, Wells CP, O'Doherty MJ, Collins RE. Tc-99m sestamibi: a new agent for parathyroid imaging. Nucl Med Commun. 1989;10:791-794.

2. Bergenfelz A, Tennvall J, Valdermarsson S, Lindblom P, Tibblin S. Sestamibi versus thallium subtraction scintigraphy in parathyroid localization: a prospective comparative study in patients with predominantly mild primary hyperparathyroidism. Surgery. 1997;121:601-605.

3. Palestro CJ, Tomas MB, Tronco GG. Radionuclide imaging of the parathyroid glands. Semin Nucl Med. 2005;35:266-276.

4. Kipper MS, Labarbera JJ, Krohn LD, Marcou M. Localization of a parathyroid adenoma by the addition of pinhole image to Tc-99m sestamibi dual-phase scintigraphy. Clin Nucl Med. 1997;22:73-75.

5. Smith JR, Oates ME. Radionuclide imaging of the parathyroid glands: patterns, pearls, and pitfalls. Radiographics. 2004;24:1101-1115.

6. Tomas MB, Pugliese P, Tronco GG, Love C, Palestro CJ, Nicholas KJ. Pinhole versus parallel-hole collimators for parathyroid imaging: an intra-individual comparison. J Nucl Med Technol. 2008;36:189-194.

7. Ho Shon IA, Bernard EJ, Roach PJ, Delbridge LW. The value of oblique pinhole images in pre-operative localization with ${ }^{99 \mathrm{~m} T c-M I B I}$ for primary hyperparathyroidism. Eur J Nucl Med. 2001;28:736-742.

8. Ho Shon IA, Yan W, Roach PJ, et al. Comparison of pinhole and SPECT ${ }^{99 \mathrm{~m} T c-}$ MIBI imaging in primary hyperparathyroidism. Nucl Med Commun. 2008;29: 949-955.

9. Nichols KJ, Tomas MB, Tronco GG, et al. Preoperative parathyroid scintigraphic lesion localization: accuracy of various types of readings. Radiology. 2008;248: 221-232. 
10. Spanu A, Falchi A, Manca A, et al. The usefulness of neck pinhole SPECT as a complementary tool to planar scintigraphy in primary and secondary hyperparathyroidism. J Nucl Med. 2004;45:40-48.

11. Arveschoug AK, Bertelsen H, Vammen B. Presurgical localization of abnormal parathyroid glands using a single injection of Tc-99m sestamibi: comparison of high-resolution parallel-hole and pinhole collimators, and interobserver and intraobserver variation. Clin Nucl Med. 2002;27:249-254.

12. Civelek AC, Ozalp E, Donovan P, Udelsman R. Prospective evaluation of delayed technetium-99m sestamibi SPECT scintigraphy for preoperative localization of primary hyperparathyroidism. Surgery. 2002;131:149157.

13. Ruda JM, Hollenbeak CS, Stack BC. A systematic review of the diagnosis and treatment of primary hyperparathyroidism from 1995 to 2003. Otolaryngol Head Neck Surg. 2005;132:359-372.
14. Kwan T-H, Ma K-M, Yung C-Y, Au T-C. Possible roles of MIBI parathyroid scan in assessment of dialysis patients with hyperparathyroidism. Hong Kong $J$ Nephrol. 2000;2:32-38.

15. Billotey C, Sarfati E, Aurengo A, et al. Advantages of SPECT in Tc-99m sestamibi parathyroid scintigraphy. J Nucl Med. 1996;37:1773-1778.

16. Slater A, Gleeson FV. Increased sensitivity and confidence of SPECT over planar imaging in dual-phase sestamibi for parathyroid adenoma detection. Clin Nucl Med. 2005;30:1-3.

17. Lavely WC, Goetze S, Friedman KP, et al. Comparison of SPECT/CT, SPECT, and planar imaging with single- and dual-phase ${ }^{99 \mathrm{~m}} \mathrm{Tc}$-sestamibi parathyroid scintigraphy. J Nucl Med. 2007;48:1084-1089.

18. Gayed IW, Kim EE, Broussard WF, et al. The value of ${ }^{99 \mathrm{~m} T c-s e s t a m i b i ~ S P E C T /}$ CT over conventional SPECT in the evaluation of parathyroid adenomas or hyperplasia. J Nucl Med. 2005;46:248-252. 